\title{
Unifying the flavor origin of dark matter with leptonic nonzero $\theta_{13}$
}

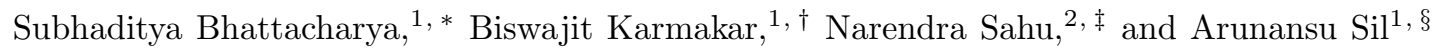 \\ ${ }^{1}$ Department of Physics, Indian Institute of Technology Guwahati, Assam-781039, India \\ ${ }^{2}$ Department of Physics, Indian Institute of Technology Hyderabad, Yeddumailaram, 502205, Telengana, India
}

We propose a flavor symmetric approach to unify the origin of dark matter (DM) with the nonzero $\theta_{13}$ in the lepton sector. In this framework, breaking of a $U(1)$ flavor symmetry at high scale into a remnant $Z_{2}$ ensures the stability of the DM and gives rise to a modification to the existing $A_{4}$-based tri-bimaximal neutrino mixing to acquire required non-zero $\sin \theta_{13}$. This results in a range of Higgs portal coupling of the DM which can be potentially accessible at various ongoing and future direct and collider search experiments.

Flavor symmetries play important roles in understanding many issues in particle physics including quark and lepton mixing as well as mass hierarchies. Historically a global $U(1)$ flavor symmetry was proposed to explain the quark mass hierarchy and Cabibbo mixing angle [1] which was extended to explain neutrino masses and mixing later. Among others, a tri-bimaximal (TBM) lepton mixing generated from a discrete flavor symmetry such as $A_{4}$ gets particular attention [2, 3, due to its simplicity and predictive nature. However, TBM mixing primarily is associated with a vanishing reactor mixing angle $\theta_{13}$ which is against the recent robust observation of non-zero $\theta_{13} \approx 9^{\circ}$ [4] by DOUBLE CHOOZ [7], Daya Bay [8], RENO [9] and T2K [10] experiments. Hence, an alteration to TBM structure has been under scanner.

Understanding the nature of dark matter (DM) is another outstanding problem in particle physics today. Although astrophysical evidences, such as rotation curves of galaxies, gravitational lensing and large scale structure of the Universe supports the existence of DM [11, a discovery in laboratory is still awaited. The relic abundance of DM has been measured by WMAP 12 and PLANCK 13] satellite experiments to be about $26.8 \%$ of the total energy budget of the Universe. Although this hints towards a broad classification of DM scenarios, its properties apart from gravitational interactions, are not known yet.

In this letter we propose a $U(1)$ flavor extension of the Standard Model (SM) to unify the origin of DM with the simultaneous realization of non-zero $\sin \theta_{13}$ in the lepton sector. For this purpose, we presume the existence of a TBM neutrino mixing pattern (in a basis where charged leptons are diagonal) and vector like fermions in the dark sector. We will argue that this serves as a minimal extension to SM to accommodate DM and non-zero $\sin \theta_{13}$. A flavon field charged under this $U(1)$ plays the role of a messenger between the DM and neutrino sector. The $U(1)$ symmetry, once allowed to be broken by the vacuum expectation value (vev) of this flavon, generates a non-zero $\sin \theta_{13}$ when the electroweak symmetry

\footnotetext{
*Electronic address: subhab@iitg.ernet.in

$\dagger$ Electronic address: k.biswajit@iitg.ernet.in

‡Electronic address: nsahu@iith.ac.in

$\S$ Electronic address: asil@iitg.ernet.in
}

is also broken. Simultaneously the interaction with dark sector leads to an effective Higgs portal coupling of the vector-like fermionic DM which yields correct relic density and can be verified at future direct search experiments, such as Xenon1T [14] and at the Large Hadron Collider (LHC) [15, 16.

We consider an effective field theory approach for the demonstration purpose and begin by assuming a typical well known structure of the neutrino mass matrix [2, 3], $\left(m_{\nu}\right)_{0}$, given by

$$
\left(m_{\nu}\right)_{0}=\left(\begin{array}{ccc}
a-2 b / 3 & b / 3 & b / 3 \\
b / 3 & -2 b / 3 & a+b / 3 \\
b / 3 & a+b / 3 & -2 b / 3
\end{array}\right),
$$

which results in a TBM neutrino mixing pattern while the charged lepton mass matrix is diagonal. The TBM mixing matrix [17] can be represented by:

$$
U_{T B M}=\left(\begin{array}{ccc}
\sqrt{\frac{2}{3}} & \frac{1}{\sqrt{3}} & 0 \\
-\frac{1}{\sqrt{6}} & \frac{1}{\sqrt{3}} & -\frac{1}{\sqrt{2}} \\
-\frac{1}{\sqrt{6}} & \frac{1}{\sqrt{3}} & \frac{1}{\sqrt{2}}
\end{array}\right)
$$

implying $\sin \theta_{13}=0, \sin ^{2} \theta_{12}=1 / 3$ and $\sin ^{2} \theta_{23}=$ $1 / 2$. The above structure of $\left(m_{\nu}\right)_{0}$ can be obtained in a $A_{4}$ based set-up either in a type-I or II see-saw framework 18 20 or through higher dimensional lepton number violating operators. For example, we can have Altarelli-Feruglio (AF) model [3, where the SM doublet leptons $(\ell)$ are transforming as triplet under the $A_{4}$ while the singlet charged leptons $e_{R}, \mu_{R}$ and $\tau_{R}$ transform as $1,1^{\prime \prime}$ and $1^{\prime}$ respectively. Then a higher dimensional operator of the form, $(\ell H \ell H)\left(\xi-y \phi_{S}\right) / \Lambda^{2}$ can be considered, where $\xi$ and $\phi_{S}$ are singlet and triplet flavon fields (they are SM singlet and transform under $A_{4}$ ) respectively. $\Lambda$ is the cut off scale of the theory and $y$ represents the relative strength between the two couplings involved. Once these flavons get vevs, a flavor structure can be generated after electroweak symmetry breaking with $a=\left(v^{2} / \Lambda\right) \epsilon$ and $b=y\left(v^{2} / \Lambda\right) \epsilon$ where $\epsilon=\langle\xi\rangle / \Lambda=\left\langle\phi_{S}\right\rangle / \Lambda$. With a judicious choice of additional discrete symmetries like $Z_{3}$ or more, one can ensure that no other terms involving these flavons and SM fields are allowed at $1 / \Lambda^{2}$ order or below so as to keep the structure of $\left(m_{\nu}\right)_{0}$ intact as in Eq. (1). In what follows, we introduce an additional global $U(1)$ flavor symmetry which will be broken into a remnant $Z_{2}$ and an additional 
contribution to $\left(m_{\nu}\right)_{0}$ becomes functional. None of the fields in the above dimension- 6 operators, responsible for TBM mixing, would carry any $U(1)$ charge in order to generate non-zero $\sin \theta_{13}$ and establish a connection to the dark sector.

Scalar singlet extension to SM as DM suffers from availability of a tiny allowed parameter space after nonobservation in direct and collider search experiments 21 . In a venture to accommodate fermionic DM with a minimal extension to the SM, we consider the dark sector to be described by a vector-like $S U(2)_{L}$ doublet fermion $\psi^{T}=\left(\psi^{0}, \psi^{-}\right)$and a vector-like neutral singlet fermion $\chi^{0}[15$. These fermions are charged under the additional $U(1)$ flavor symmetry, but neutral under the existing symmetry in the neutrino sector (say the non-abelian $A_{4}$ and additional discrete symmetries required). We also introduce two other SM singlet flavon fields $\phi$ and $\eta$ which carry equal and opposite charges under the $U(1)$ symmetry but transform as 1 and $1^{\prime}$ under $A_{4}$. Note that the SM fields are neutral under this additional $U(1)$ symmetry. The effective Lagrangian, invariant under the symmetries considered, describing the interaction between the dark and the SM sector is then given by:

$$
\mathcal{L}_{\text {int }}=\left(\frac{\phi}{\Lambda}\right)^{n} \bar{\psi} \widetilde{H} \chi^{0}+\frac{(\ell H \ell H) \phi \eta}{\Lambda^{3}}
$$

We keep $n$ as a free parameter at present. The first term is allowed since the $U(1)$ charge of $\phi^{n}$ is compensated by $\psi$ and $\chi^{0}$, while the second term is allowed since the $U(1)$ charges of $\phi$ and $\eta$ cancel with each other. This also ensures that $\phi$ and $\eta$ do not take part in $\left(m_{\nu}\right)_{0}$. The detailed structure of the scenario is left for a future work [22. The idea of introducing a vector like fermion in the dark sector is also motivated by the fact that we expect a replication of the SM Yukawa type interaction to be present in the dark sector as well. Here the $\phi$ field plays the role of the messenger field similar to the one considered in 23 .

When $\phi$ and $\eta$ acquire vevs, the $U(1)$ symmetry breaks into a remnant $Z_{2}$ symmetry under which the vectorlike fermions $\psi$ and $\chi^{0}$ are odd. Consequently the DM emerges as an admixture of the neutral component of the vector-like fermions $\psi$ and $\chi^{0}$ and yields a larger region of allowed parameter space as we will shortly demonstrate. The interaction strength of the DM with the SM Higgs is then given by $(\langle\phi\rangle / \Lambda)^{n} \equiv \epsilon^{n}$. Similarly the second term in Eq. (3) provides an additional contribution to the light neutrino mass matrix as follows:

$$
\delta m_{\nu}=\left(\begin{array}{ccc}
0 & 0 & d \\
0 & d & 0 \\
d & 0 & 0
\end{array}\right)
$$

where $d=\left(v^{2} / \Lambda\right) \epsilon^{2}$ with $\epsilon=\langle\phi\rangle / \Lambda \equiv\langle\eta\rangle / \Lambda$. This typical flavor structure follows from the involvement of $\eta$ field, which transforms as $1^{\prime}$ under $A_{4}[24$.

From Eqs. (1) and (4), we get the light neutrino mass matrix as $m_{\nu}=\left(m_{\nu}\right)_{0}+\delta m_{\nu}$. We have already seen that the $\left(m_{\nu}\right)_{0}$ can be diagonalized by $U_{T B M}$ alone, so an additional rotation $\left(U_{1}\right)$ is required to diagonalize $m_{\nu}$ :

$$
U_{1}=\left(\begin{array}{ccc}
\cos \theta_{\nu} & 0 & \sin \theta_{\nu} \\
0 & 1 & 0 \\
-\sin \theta_{\nu} & 0 & \cos \theta_{\nu}
\end{array}\right) .
$$

Here we assume all parameters $a, b, d$ are real for simplicity. We therefore obtain

$$
\tan 2 \theta_{\nu}=\frac{\sqrt{3} d}{d-2 a}=\frac{\sqrt{3} \epsilon}{\epsilon-2}
$$

Then comparing the standard $U_{P M N S}$ parametrization and neutrino mixing matrix $U_{\nu}\left(=U_{T B M} U_{1}\right)$ we get $\sin \theta_{13}=\sqrt{\frac{2}{3}}\left|\sin \theta_{\nu}\right|, \sin ^{2} \theta_{12}=\frac{1}{3\left(1-\sin ^{2} \theta_{13}\right)}, \sin ^{2} \theta_{23}=$ $\frac{1}{2}+\frac{1}{\sqrt{2}} \sin \theta_{13}, \delta=\arg \left[\left(U_{1}\right)_{13}\right]=0$.

Clearly $\sin \theta_{13}$ depends only on $\epsilon$ as shown in Fig 1 The horizontal patch in Fig 1 denotes the allowed $3 \sigma$ range of $\sin \theta_{13}$ (三0.1330-0.1715) [6] which is in turn restrict the range of $\epsilon$ parameter denoted by the vertical patch on the figure. Note that the interaction strength of DM with the SM particles depend on $\epsilon^{n}$. Therefore we find that the size of $\sin \theta_{13}$ is intimately related with the Higgs portal coupling of DM. This is an important observation in this letter and is demonstrated in the rest of the paper. The two other mixing angles $\theta_{12}$ and $\theta_{23}$ fall in the right ballpark while light neutrino mass satisfy the $\Delta m_{\odot}^{2}=7.60 \times 10^{-5} \mathrm{eV}^{2}$ and $\left|\Delta m_{\text {atm }}^{2}\right|=2.48 \times 10^{-3}$ $\mathrm{eV}^{2}$ [4] 6 .

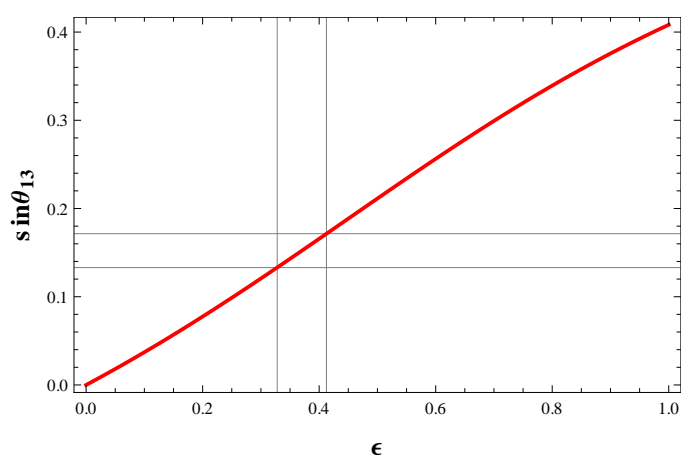

FIG. 1: Plot of $\sin \theta_{13}$ against $\epsilon$. Here $3 \sigma$ range [6] for $\sin \theta_{13}$ fixes $\epsilon$ in the range $0.328-0.4125$.

Now we focus on the 1st term of Eq. (3) to estimate the relic density of dark matter as a function of $\epsilon$. Since $\psi$ and $\chi^{0}$ are vector-like fermions, they can have bare masses, $M_{\psi} \bar{\psi} \psi$ and $M_{\chi} \overline{\chi^{0}} \chi^{0}$, which are not protected by the SM symmetry. The electroweak phase transition also gives rise a mixing between $\psi^{0}$ and $\chi^{0}$. In the basis $\left(\chi^{0}, \psi^{0}\right)$, the mass matrix is given by

$$
\mathcal{M}=\left(\begin{array}{cc}
M_{\chi} & Y v \\
Y v & M_{\psi}
\end{array}\right)
$$

where $Y=\epsilon^{n}$. Diagonalizing the above mass matrix we get the mass eigenvalues as $M_{1}$ and $M_{2}$ corresponding to 
the mass eigenstates $\psi_{1}$ and $\psi_{2}$. We assume that $\psi_{1}$ is the lightest odd particle and hence constitute the DM of the Universe. The mixing angle is given by

$$
\sin 2 \theta_{d} \simeq \frac{2 Y v}{\Delta M}
$$

in the small mixing limit where $\Delta M=M_{2}-M_{1}$. Here, we note that small mixing is necessary for the model to provide a DM with viable relic density. This is because, the larger is the doublet content in DM $\psi_{1}$, the annihilation goes up significantly in particular through $\psi_{1} \overline{\psi_{1}} \rightarrow$ $W^{+} W^{-}$. So in the limit, $\psi_{2}$ is dominantly a doublet having a small admixture of the singlet one. This implies that $\psi_{2}$ mass is required to be larger than $45 \mathrm{GeV}$ in order not to conflict with the invisible $Z$-boson decay width. In the physical spectrum we also have a charged fermion $\psi^{+}\left(\psi^{-}\right)$with mass $M^{+}\left(M^{-}\right)=M_{1} \sin ^{2} \theta_{d}+M_{2} \cos ^{2} \theta_{d}$. In the limit $\theta_{d} \rightarrow 0, M^{ \pm}=M_{2}=M_{\psi}$

The relic density of the $\psi_{1}$ dark matter is mainly dictated by annihilations $\overline{\psi_{1}} \psi_{1} \rightarrow W^{+} W^{-}$and $\overline{\psi_{1}} \psi_{1} \rightarrow h h$. The other relevant channels are mainly co-annihilation of $\psi_{1}$ with $\psi_{2}$ and $\psi^{ \pm}$which dominantly contribute to relic density in a large parameter space [15, 25, 28. The dark-sector is mainly dictated by three parameters $\sin \theta_{d}, M_{1}, M_{2}$. In the following we use Micromega [29] to find the allowed region of correct relic abundance for $\psi_{1}$ DM satisfying WMAP 12 constraint ${ }^{1}$

$$
0.094 \leq \Omega_{\mathrm{DM}} h^{2} \leq 0.130 .
$$

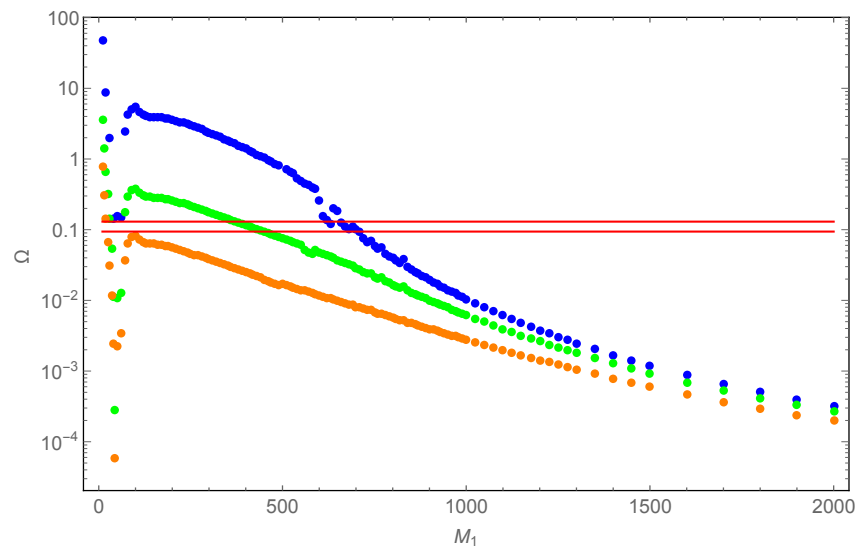

FIG. 2: Relic density vs DM mass $M_{1}$ (in $\mathrm{GeV}$ ) for different choices of $Y=0.04,0.08,0.115$ (Blue, Green, Orange respectively) with $\Delta M=100 \mathrm{GeV}$. Horizontal lines define the correct relic density.

In Fig. 2 we plot relic density versus DM mass $M_{1}$ for different choices of $Y(=0.04,0.08,0.115$ with Blue,

\footnotetext{
1 The range we use corresponds to the WMAP results; the PLANCK constraints $0.112 \leq \Omega_{\mathrm{DM}} h^{2} \leq 0.12813$, though more stringent, do not lead to significant changes in the allowed regions of parameter space.
}

Green and Orange respectively from top to bottom), keeping the mass difference $\Delta M$ fixed at $100 \mathrm{GeV}$. The mixing angle $\sin \theta_{d}$ (as obtained using Eq. (8)) associated with the top (Blue) line in 0.1 and it increases to 0.2 and 0.3 for the middle (Green) and bottom (Orange) lines. As the mixing increases, the doublet component starts to dominate and hence give larger cross-section which leads to a smaller DM abundance. Note that $\sin \theta_{d}=0.3$ $(Y=0.115)$ can barely satisfy relic density, where annihilations through $Z$ mediation becomes large. In Fig. 3 . we plot $Y$ versus $M_{1}$ to produce correct relic density with $\sin \theta_{d}=0.1$ and 0.2 while varying $\Delta M$. It points out a relatively wide DM mass range satisfy the relic density constraint. It also shows that for $\sin \theta_{d}=0.1$ (generally true for $\sin \theta_{d} \leq 0.1$ ), the annihilations are never enough to produce correct density and co-annihilations play a crucial part resulting the blue curve rising with the DM mass. For $\sin \theta_{d}=0.2$ (green patch), smaller DM mass regions get contributions from coannihilation with small $Y$ and annihilations only for large $Y$, while the region close to DM mass $400 \mathrm{GeV}$ has a significant contribution from $Z$ mediation.

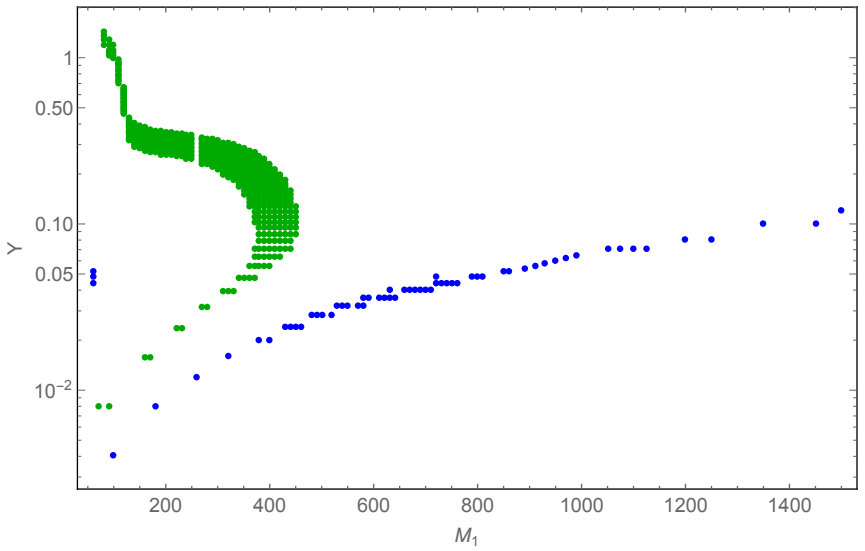

FIG. 3: $Y$ versus $M_{1}$ (in $\mathrm{GeV}$ ) for correct relic density (Eq. 9). $\sin \theta=0.1,0.2$ (Blue and Green respectively) has been chosen, while $\Delta M$ vary arbitrarily.

The most stringent constraint on the Higgs portal coupling $Y \simeq \sin 2 \theta_{d} \Delta M /(2 v)$ comes from the direct search of DM at Xenon-100 [30], LUX [31] as demonstrated in Fig.4. We see that the bound from LUX, constraints the coupling: $Y \sim 0.05$ for DM masses $\gtrsim 800 \mathrm{GeV}$ (Green points). The Yukawa coupling needs to be even smaller for $M_{1} \simeq 100 \mathrm{GeV}$. Though large couplings are allowed by correct relic density, but they are highly disfavored by the direct DM search at terrestrial experiments. Note that these constraints are less dependent on $\Delta M$ as to the mixing angle, which plays otherwise a crucial role in the relic abundance of DM.

We can now combine the outcome of the two sectors into Fig. 5. The allowed range of $Y$-values can be translated in terms of $\epsilon-n$ as shown here. Correct $\sin \theta_{13}$ allowed $\epsilon$ within $0.328-0.4125$ (see Fig. (1). Therefore, the Higgs portal couplings: $Y \lesssim 0.05$, allowed by correct 


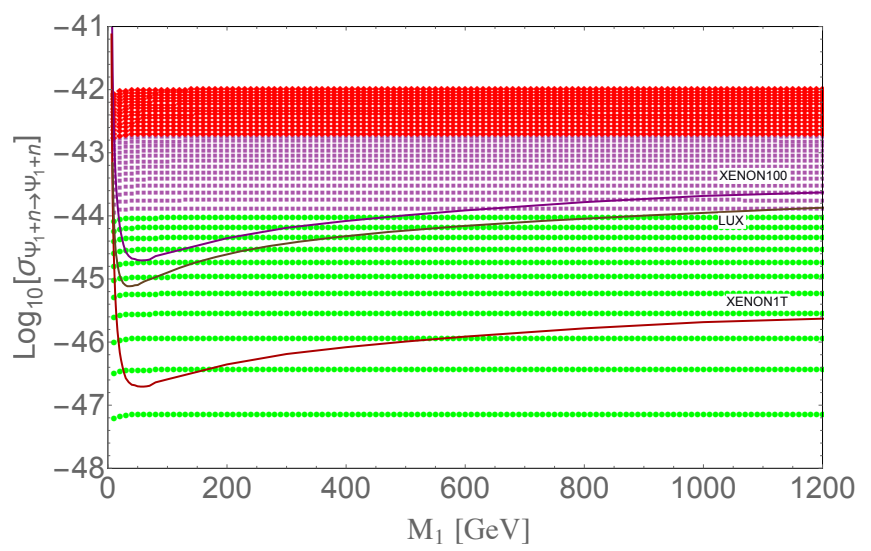

FIG. 4: Allowed values of the Higgs portal coupling $Y$ by the direct search experiments, Xenon100, LUX and Xenon1T: $Y:\{0.001-0.05\}$ (Green), $Y:\{0.05-0.1\}$ (Purple), $Y:\{0.1-0.15\}$ (Red). $\Delta M=100 \mathrm{GeV}$ is used for the scan.

relic density and direct search of DM can be satisfied with $n=3$ or more.

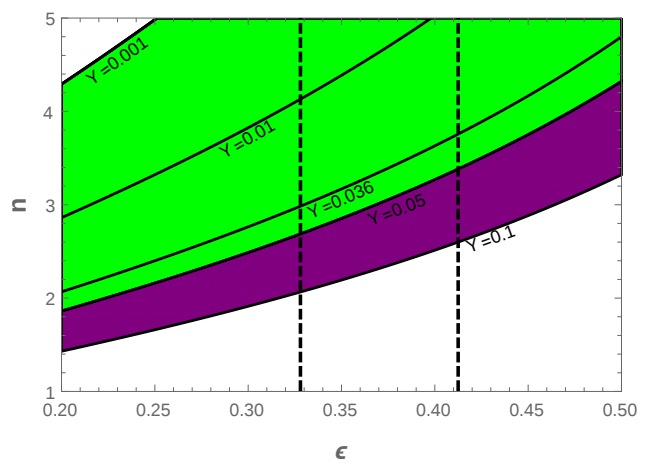

FIG. 5: $n$ vs $\epsilon$ to generate different values of $Y=\epsilon^{n}$.
The $U(1)$ symmetry of the model is broken by the vev of a flavon field to a remnant $Z_{2}$, whereas the breaking of $A_{4}$ (and additional discrete symmetry) is responsible for producing the flavor structure of neutrino mass matrix. The details of symmetry breaking pattern and charge assignment of the flavon fields is worthy of attention. Non-zero $\sin \theta_{13}$ appeals for finite values of phases and hence $\mathrm{CP}$-violations, which have been ignored in this letter. They will be discussed together in a future publication 22].

In summary, the observed value of non-zero $\sin \theta_{13}$ and its link to Higgs portal coupling of a vector-like fermionic DM was obtained in a further $U(1)$ flavor extension of the SM. We showed that the non-zero values of $\sin \theta_{13}$ fixes a range of Higgs portal coupling $Y=\epsilon^{n}, n \gtrsim 3$ which can be probed at the future direct DM search experiments such as Xenon-1T. Also note that the next to lightest stable particle (NLSP) could be a charged fermion which can be searched at the LHC [16. In the limit of small $\sin \theta_{d}$, the NLSP can give rise to a displaced vertex at LHC, a rather unique signature for the model discussed [15].

\section{ACKNOWLEDGEMENTS}

The work of SB is partially supported by DST INSPIRE grant no PHY/P/SUB/01 at IIT Guwahati. NS is partially supported by the Department of Science and Technology, Govt. of India under the financial Grant SR/FTP/PS-209/2011.
[1] C. D. Froggatt and H. B. Nielsen, Nucl. Phys. B 147, 277 (1979). doi:10.1016/0550-3213(79)90316-X

[2] E. Ma and G. Rajasekaran, Phys. Rev. D 64 (2001) 113012 doi:10.1103/PhysRevD.64.113012 [hep$\mathrm{ph} / 0106291]$.

[3] G. Altarelli and F. Feruglio, Nucl. Phys. B 741, 215 (2006) [hep-ph/0512103].

[4] F. Capozzi, G. L. Fogli, E. Lisi, A. Marrone, D. Montanino and A. Palazzo, Phys. Rev. D 89, no. 9, 093018 (2014) [arXiv:1312.2878 [hep-ph]].

[5] M. C. Gonzalez-Garcia, M. Maltoni and T. Schwetz, JHEP 1411, 052 (2014) [arXiv:1409.5439 [hep-ph]].

[6] D. V. Forero, M. Tortola and J. W. F. Valle, Phys. Rev. D 90, no. 9, 093006 (2014) [arXiv:1405.7540 [hep-ph]].

[7] Y. Abe et al. [Double Chooz Collaboration], Phys. Rev. Lett. 108, 131801 (2012) doi:10.1103/PhysRevLett.108.131801 [arXiv:1112.6353 [hep-ex]].

[8] F. P. An et al. [Daya Bay Collaboration], Phys. Rev. Lett. 108, 171803 (2012) doi:10.1103/PhysRevLett.108.171803 [arXiv:1203.1669 [hep-ex]].

[9] J. K. Ahn et al. [RENO Collaboration], Phys. Rev. Lett. 108, 191802 (2012) doi:10.1103/PhysRevLett.108.191802 [arXiv:1204.0626 [hep-ex]].

[10] K. Abe et al. [T2K Collaboration], Phys. Rev. Lett. 112, 061802 (2014) doi:10.1103/PhysRevLett.112.061802 [arXiv:1311.4750 [hep-ex]].

[11] G. Bertone, D. Hooper and J. Silk, Phys. Rept. 405, 279 (2005), arXiv:hep-ph/0404175.

[12] G. Hinshaw et al. [WMAP Collaboration], Astrophys. J. Suppl. 208, 19 (2013) [arXiv:1212.5226 [astro-ph.CO]].

[13] P. A. R. Ade et al. Planck Collaboration, Astron. Astrophys. 571, A16 (2014), arXiv:1303.5076 [astroph.CO]. 
[14] E. Aprile et al. [XENON Collaboration], [arXiv:1512.07501 [physics.ins-det]].

[15] S. Bhattacharya, N. Sahoo and N. Sahu, arXiv:1510.02760 [hep-ph].

[16] C. Arina, R. N. Mohapatra and N. Sahu, Phys. Lett. B 720 (2013) 130 doi:10.1016/j.physletb.2013.01.059 [arXiv:1211.0435 [hep-ph]]; C. Arina, J. O. Gong and N. Sahu, Nucl. Phys. B 865, 430 (2012) doi:10.1016/j.nuclphysb.2012.07.029 [arXiv:1206.0009 [hep-ph]].

[17] P. F. Harrison, D. H. Perkins and W. G. Scott, Phys. Lett. B 458, 79 (1999) [hep-ph/9904297].

[18] B. Karmakar and A. Sil, Phys. Rev. D 91, 013004 (2015) [arXiv:1407.5826 [hep-ph]] and references there in.

[19] G. C. Branco, R. Gonzalez Felipe, F. R. Joaquim and H. Serodio, Phys. Rev. D 86, 076008 (2012) [arXiv:1203.2646 [hep-ph]] and references there in.

[20] B. Karmakar and A. Sil, Phys. Rev. D 93, no. 1, 013006 (2016) doi:10.1103/PhysRevD.93.013006 [arXiv: 1509.07090 [hep-ph]].

[21] J. M. Cline, K. Kainulainen, P. Scott and C. Weniger, Phys. Rev. D 88, 055025 (2013) Erratum: [Phys. Rev. D 92, no. 3, 039906 (2015)] doi:10.1103/PhysRevD.92.039906, 10.1103/PhysRevD.88.055025 [arXiv:1306.4710 [hep-ph]].

[22] S. Bhattacharya, B. Karmakar, N. Sahu and A. Sil, in preparation.

[23] L. Calibbi, A. Crivellin and B. Zaldvar, Phys. Rev. D 92, no. 1, 016004 (2015) doi:10.1103/PhysRevD.92.016004 [arXiv:1501.07268 [hep-ph]].

[24] Y. Shimizu, M. Tanimoto and A. Watanabe, Prog. Theor. Phys. 126, 81 (2011) doi:10.1143/PTP.126.81 [arXiv:1105.2929 [hep-ph]].

[25] K. Griest and D. Seckel, Phys. Rev. D 43, 3191 (1991)

[26] G. Cynolter and E. Lendvai, Eur. Phys. J. C 58, 463 (2008) doi:10.1140/epjc/s10052-008-0771-7 [arXiv:0804.4080 [hep-ph]].

[27] T. Cohen, J. Kearney, A. Pierce and D. TuckerSmith, Phys. Rev. D 85, $075003 \quad$ (2012) doi:10.1103/PhysRevD.85.075003 [arXiv:1109.2604 [hep-ph]].

[28] C. Cheung and D. Sanford, JCAP 1402, 011 (2014) doi:10.1088/1475-7516/2014/02/011 [arXiv:1311.5896 [hep-ph]].

[29] G. Belanger, F. Boudjema, A. Pukhov and A. Semenov, Comput. Phys. Commun. 180, 747 (2009) doi:10.1016/j.cpc.2008.11.019 [arXiv:0803.2360 [hep$\mathrm{ph}]$.

[30] E. Aprile et al. [XENON100 Collaboration], Phys. Rev. Lett. 109, 181301 (2012) doi:10.1103/PhysRevLett.109.181301 [arXiv:1207.5988 [astro-ph.CO]].

[31] D. S. Akerib et al. [LUX Collaboration], Phys. Rev. Lett. 112, 091303 (2014) doi:10.1103/PhysRevLett.112.091303 [arXiv:1310.8214 [astro-ph.CO]]. 